\title{
Chronic Diseases: Are they Mostly Caused by Oxidation or Nitration, Nitrosylation or Nitrosation (The 3N's)? Why is Chronic Disease Treatment so Inadequate?
}

\author{
Knox Van Dyke* \\ Department of Biochemistry and Molecular Pharmacology, West Virginia University Medical School, USA
}

Submission: March 25, 2017; Published: May 05, 2017

*Corresponding authors: Knox Van Dyke, Department of Biochemistry and Molecular Pharmacology, West Virginia University Medical School, USA, Email: kvandyke@hsc.wvu.edu

\section{Abstract}

The literature is replete with the idea that oxidants are the cause of most chronic diseases. But when antioxidants are used to scavenge these oxidants in chronic diseases, for the most part ineffectiveness or even an increase in toxicity has been shown in some instances. There is some evidence that antioxidants are useful in protection of certain eye diseases. This should not be surprising since the eyes are constantly bombarded by light which creates an oxidative condition for a major portion of the day. Why can't we treat chronic inflammation effectively?

\section{Acute Inflammation}

Unfortunately, oxidation is used to encompass both oxidation and the $3 \mathrm{~N}^{\prime} \mathrm{s}$. This is a fundamental chemical or biological mistake. The cause of chronic diseases is for the most part chronic inflammation and it is caused by the 3N's.

Acute inflammation is characterized by activation of the transcription factor nf-kappa $\mathrm{b}$ and acetylation of histone proteins at the epsilon amino groups of lysines in transcriptomes of inflammatory cells. DNA is negatively charged due to its phosphate groups and the DNA helix is wrapped around spherical inflammasomes/histones. When the histones are acetylated by the enzyme histone acetyl transferase (HAT)-this causes the positive charge to be neutralized. This opens up the sites on the promoters of inflammatory genes - so they can be continuously transcribed. Therefore, the inflammatory process continues to produce a multitude of inflammatory products developed in acute inflammation. In addition, macrophages produce an increased amount of a small amount of a peroxide called peroxynitrite. When steroidal anti-inflammatory drugs are used for treatment - a stimulation of enzymatic activity of histone deacetylase (HDAC-2) occurs and the acetate groups are removed and the DNA binds tightly to the histones and inflammation ceases.

\section{Chronic Inflammation}

When acute inflammation continues to occur over a prolonged period of time- macrophages flood the area and produce excessive amounts of peroxynitrite (OONO-).

This causes nitration of histone deacetylase which poisons the histone deacetylase. Excessive peroxynitrite keeps inflammation going at a damaging pace causing surrounding cells to die and scarring to occur (fibrosis) which prevents key cells from doing their primary job. Since chronic inflammation is certainly triggered by excessive peroxynitrite production it would be prudent to control its excessive output in order to prevent damage to DNA, RNA, proteins or enzymes and lipids which cause apoptosis or necrosis types of cell death.

Peroxynitrite $(\mathrm{OON}=\mathrm{O}-)$ is a peroxide used by macrophages to fight infections from virus, bacteria, fungus, and parasites. Unfortunately, it is also produced in excess in chronic diseases in a continuous manner. It causes nitration and oxidation but it is nitration which can create much of the irreversible damage. Particularly, nitration of amino acids like tyrosines (HDAC-2) which can be found in numerous hormones or their receptors (eg. insulin and its receptors).

Peroxynitrite is produced when the two free radicals of nitric oxide (.NO) and superoxide (.O2-) react together at 
diffusional speed. A logical method to destroy peroxynitrite would be to destroy either superoxide or nitric oxide but the side effects of this maneuver would be devastating. Peroxynitrite can be controlled by using a mixture of nitration targets The idea is to use substances that are non-toxic targets of nitration. Particularly, substances like omega 3 fatty acids, azaxanthin, L-tyrosine, many of the forms of tocopherols or tocotrienolsie vitamin $\mathrm{E}$ congeners and sustained release vitamin $\mathrm{C}$ and/or sustained release inosine.

A fundamental problem involved with treating chronic diseases is to treat early enough in the acute inflammatory disease so it does not have the chance to become very chronic and hence mostly untreatable. But how could it be learned when to start early treatment. Chronic inflammation can be found when a sample of blood is drawn and the white cells separated from the red cells. The white cell pellet is lysed (broken) and centrifuged and then subjected to SDS gel electrophoresis. The proteins are separated. The proteins are electroblotted on to a membrane, washed and primary antibody to nitrated tyrosines is applied, washed, secondary antibody that binds to the first antibody with a horse radish peroxidase label is bound and enhance luminescence is assayed for its concentration. Light is proportional to amount of nitrated tyrosines. Another method is to prevent excessive nitration is by taking the correct supplements or drugs before chronic inflammation occurs."An ounce of prevention is worth more than a pound of cure", said Benjamin Franklin. 